\title{
Comparação entre três técnicas coproparasitológicas na investigação de parasitos intestinais de seres humanos
}

\author{
Comparison between three coproparasitological techniques in the investigation of \\ intestinal parasites in humans
}

Comparación entre tres técnicas coproparasitológicas en la investigación de parásitos

intestinales en humanos

Karina Rodrigues dos Santos ${ }^{1 *}$, Eduardo Rodrigues Ciro ${ }^{1}$, Luanny da Silva Rodrigues Miranda ${ }^{1}$, Márcia Nogueira Lino ${ }^{1}$, Severino Cavalcante de Sousa Júnior ${ }^{1}$.

\section{RESUMO}

Objetivo: Este trabalho tem como objetivo, testar e comparar três técnicas coproparasitológicas para o diagnóstico de parasitos intestinais de seres humanos. Métodos: Foram realizadas 141 análises de amostras fecais de pessoas com variadas idades, utilizando três metodologias: método de Hoffman, Pons e Janeradaptado por Katagiri S e Oliveira-sequeira TCG (2007), método de Willis-Mollay (1921) e a técnica de Gordon e Whitlock-modificada (ou OPG). Resultados: A prevalência de helmintíases utilizando o método de Hoffman, Pons e Janer-adaptado foi de 14,18\% (20), para o método de Willis-Mollay foi de 15,32\% (19) e para a técnica de OPG foi de 12,94\% (15). Conclusão: Os resultados mostraram que o método de Willis-Mollay recuperou um número maior de gêneros de parasitos instestinais e demonstrou ser mais rápido e de fácil execução. $\mathrm{E}$ com a realização das três metodologias, pode-se observar que as espécies de Ascaris lumbricoides e Trichuris trichiura foram às de maior prevalência neste estudo.

Palavras-chave: Técnicas parasitológicas, Parasitologia, Helmintos.

\begin{abstract}
Objective: This work aims to test and compare three coproparasitological techniques for the diagnosis of intestinal parasites in humans. Methods: 141 analyzes of faecal samples from people of varying ages were carried out, using three methodologies: the method of Hoffman, Pons and Janer-adapted by Katagiri $S$ and Oliveira-sequeira TCG (2007), the method of Willis-Mollay (1921) and the technique of Gordon and Whitlockmodified (or OPG). Results: he prevalence of helminthiasis using the Hoffman, Pons and Janer-adapted method was $14.18 \%$ (20), for the Willis-Mollay method it was $15.32 \%$ (19) and for the OPG technique it was $12,94 \%$ (15). Conclusion: The results showed that the Willis-Mollay method recovered a greater number of genera of instestinal parasites and proved to be faster and easier to perform. And with the realization of the three methodologies, it can be observed that the species of Ascaris lumbricoides and Trichuris trichiura were the most prevalent in this study.
\end{abstract}

Keywords: Parasitological techniques, Parasitology, Helminth.

\section{RESUMEN}

Objetivo: Este trabajo tiene como objetivo probar y comparar tres técnicas coproparasitológicas para el diagnóstico de parásitos intestinales en humanos. Métodos: Se realizaron 141 análisis de muestras fecales

1 Universidade Federal do Delta do Parnaíba (UFDPar). Parnaíba - PI.

*E-mail: krsantos2004@yahoo.com.br

SUBMETIDO EM: 12/2019

ACEITO EM: 1/2020

PUBLICADO EM: 7/2020

REAS/EJCH | Vol.Sup.n.52 | e3521 | DOI: https://doi.org/10.25248/reas.e3521.2020 Página 1 de 9 
de personas de diferentes edades, utilizando tres metodologías: el método de Hoffman, Pons y Janer, adaptado por Katagiri S y Oliveira-sequeira TCG (2007), el método de Willis-Mollay (1921) y la técnica de Gordon y Whitlock modificado (u OPG). Resultados: La prevalencia de helmintiasis con el método adaptado de Hoffman, Pons y Janer fue del $14.18 \%$ (20), para el método Willis-Mollay fue del $15.32 \%$ (19) y para la técnica OPG fue 12 , 94\% (15). Conclusión: Los resultados mostraron que el método Willis-Mollay recuperó un mayor número de géneros de parásitos instestinales y demostró ser más rápido y fácil de realizar. Y con la realización de las tres metodologías, se puede observar que las especies de Ascaris lumbricoides y Trichuris trichiura fueron las más prevalentes en este estudio.

Palabras clave: Técnicas paras conclusión parasitológicas, Parasitología, Helmintos.

\section{INTRODUÇÃO}

As doenças infecciosas e parasitárias representam cerca de duas a três milhões de mortes anualmente no mundo, principalmente nos países em desenvolvimento, devido as precárias condições de higiene dos indivíduos de baixa renda, que moram em locais com aglomeração intensa de pessoas e vivendo em condições de saneamento e coleta de lixo inadequadas, o que favorecem as altas taxas de contaminação da água e dos alimentos consumidos, favorecendo à transmissão de diversos microrganismos (MELO AFL, et al., 2014; ALEXANDRE TS, et al., 2015; VASCONCELOS IAB, et al., 2011). A transmissão das doenças parasitárias ocorre, principalmente, pela via oral-fecal, pela forma infectante do parasito. Todavia, existem outras vias de transmissão dessas parasitoses, como a percutânea, onde o indivíduo é contaminado, na maioria das vezes, pelo hábito de andar descalço (ALVES FA, et al., 2014).

Os parasitos intestinais estão entre os agentes patogênicos mais comumente encontrados em seres humanos e animais de companhia. Os parasitos de animais domésticos mais comuns e com potencial zoonótico, são os protozoários dos gêneros: Giardia, Toxoplasma e Cryptosporidium e dentre os helmintos, principalmente os gêneros Toxocara spp. e Ancylostoma spp. (MONTEIRO SG, 2010). Dos nematódeos específicos do homem, os mais prevalentes são: Ascaris lumbricoides, Ancylostoma duodenale, Trichuris trichiura, Enterobius vermicularis, Hymenolepis nana e Strongyloides stercoralis (FERREIRA MU, 2012).

Os indivíduos mais susceptíveis a essas doenças são as crianças, uma vez que frequentam ambientes coletivos, que possibilitam as constantes infecções e reinfecções, por estarem constantemente contato com o solo, além de apresentarem sistema imunológico imaturo (ALVES FA, et al., 2014; ALEXANDRE TS, et al., 2015; NUNES XP, et al., 2014).

As crianças quando parasitadas, podem apresentar sintomas de diarréia, anemia, obstrução intestinal, desnutrição e quando são acometidas por parasitos percutâneos podem apresentar dermatites cutáneas e todas estas parasitoses podem levar a graves consequências como: alterações no estado físico, psicossomático e social, que interferem diretamente na qualidade de vida dessas crianças (SANTOS KR, et al., 2019; CARDOSO AS, et al., 2018; BELO VS et al., 2012; VASCONCELOS IAB et al., 2011).

Para o diagnóstico de infecções por parasitos intestinais podem ser empregadas diferentes técnicas parasitológicas para a pesquisa de helmintos intestinais, que devem ser escolhidas conforme histórico do paciente, dados epidemiológicos e a suspeita diagnóstica (FERNANDES FC e BARSOSA FH, 2011). Mediante estas informações acima, é importante conhecer os principais métodos empregados nas rotinas clínicas laboratoriais, dentre estes podem ser empregados os exames diretos que é descrito como um método simples e rápido, porém apresenta baixa sensibilidade (FERREIRA UM, 2012).

O método de Hoffman, Pons e Janer, é atualmente o método de sedimentação por força gravitacional utilizado com maior frequência nas rotinas laboratoriais humanas no Brasil, e mais eficiente na detecção de ovos pesados como: ovos de cestódeos (Taenia spp.) e trematódeos (Schistosoma spp.), e alguns laboratórios a utilizam também para a observação de ovos leves como: Trichuris trichiura, Ancylostoma duodenale e muitas vezes até mesmo para a detecção de Giardia spp. é um método de execução simples, não alterar a viabilidade dos ovos, porém é uma técnica que necessita de um tempo maior para a sua 
execução e leitura e acumula muitos detritos dificultando a observação e identificação dos parasitos (FERREIRA MU, 2012; GONÇALVES GS, et al., 2016; KATAGIRI S e OLIVEIRA-SEQUEIRA TCG, 2007). O método de Hoffman adaptado por Katagiri S e Oliveira-Sequeira TCG (2010), uniu duas técnica em uma só: a de Hoffman, Pons e Janer e o método de Faust e se diferencia da técnica de sedimentação convencional, pois é utilizada uma centrifuga para acelerar o procedimento. Nesta os parasitos apresentam melhor visibilidade quando comparada a técnica de Hoffman, Pons e Janer, é mais rápida porque o tempo de execução é menor e leitura da lâmina é facilitada por diminuir a quantidade de detritos (KATAGIRI $S$ e OLIVEIRA-SEQUEIRA TCG, 2007).

A técnica de Willis-Mollay é empregada na rotina dos laboratórios de Análises Clínicas, sendo uma técnica qualitativa de flutuação espontânea simples, com o emprego de uma solução de elevada densidade (1:1200), o principio desta é fazer com que os ovos de menor densidade flutuem, aderindo a superfície inferior da lâmina (HOFFMAN RP, 1987; FERREIRA MU, 2012).

A técnica de Gordon e Whitlock modificado é uma técnica de flutuação, sendo utilizada para o diagnóstico quantitativo dos parasitos que causam doenças em animais, principalmente ruminantes (HOFFMAN RP, 1987). FERREIRA MU (2012) cita como uma alternativa uma diluição semelhante à utilizada na técnica de Gordon e Whitlock modificado, para diagnóstico parasitológico em humanos.

A técnica de Kato Katz é uma técnica quantitativa específica para a quantificação dos ovos das fezes de seres humanos (CHIEFFI PP, et al., 1981; FERREIRA MU, 2012). Esta necessita de uma placa ou cartão perfurado para medir o diâmetro da amostra ou kits ou reagentes para o seu preparo (CHIEFFI PP, et al., 1981; FERREIRA MU, 2012). Com o intuito de propor alternativas de métodos que apresentem metodologias mais simples, este trabalho tem como objetivo, testar e comparar três técnicas coproparasitológicas para o diagnóstico de parasitos intestinais de seres humanos.

\section{MÉTODOS}

A pesquisa foi realizada no município de Parnaíba, localizado no norte do Piauí, a uma distância de 340 $\mathrm{km}$ da capital Teresina. A cidade apresenta clima tropical, possui uma área de aproximadamente $436 \mathrm{~km}^{2} \mathrm{e}$ um total de população estimada em 149.803 (IBGE, 2015). A cidade apresenta a proporção de $22,35 \%$ dos domicílios particulares permanentes com saneamento considerado adequado, isto é, abastecimento de água por rede geral, esgotamento sanitário por rede geral ou fossa séptica e lixo coletado diretamente ou indiretamente (IBGE, 2010).

Participaram da pesquisa 60 usuários de duas Unidades Básicas de Saúde (UBS), 33 usuários da UBS Mod. 45 e 27 usuários da UBS Mod. 14 da cidade de Parnaíba, Piauí. Foram analisadas 141 amostras cedidas pelos 93 usuários e familiares. Estas amostras foram enviadas para o laboratório de Parasitologia da Universidade Federal do Delta do Parnaíba (UFDPar). Dados dessas amostras e dos doadores foram identificados e anotados em fichas individuais. As fezes quando coletadas eram acondicionadas em recipientes universais e em um isopor refrigerado e transportadas até o laboratório.

Os exames foram realizados utilizando o método de Hoffman, Pons e Janer (concentração por centrífugosedimentação) adaptado por Katagiri S e Oliveira-Sequeira TCG (2010), método de Willis-Mollay (flutuação espontânea simples) (Ferreira MU, 2012; Hoffman RP, 1987) e método de Gordon e Whitlock modificado (ou OPG - ovos por gramas de fezes) (HOFFMAN RP, 1987). O método de Hoffman adaptado por Katagiri S e Oliveira-Sequeira TCG (2010), é uma técnica qualitativa de concentração por centrífugo-sedimentação, com adaptações nos protocolos de duas técnicas: de Hoffman, Pons \& Janer e de Faust e colaboradores.

Para esta, dilui-se amostras de fezes frescas em água corrente e depois de filtradas são transferidas para um tubo de centrífuga. Após a centrifugação o líquido sobrenadante é descartado, o sedimento é homogeneizado em água corrente e novamente centrifugado. Após a homogeneização do sedimento uma ou duas gotas do material pode ser transferida para uma lâmina de microscopia e coberta com lamínula 22 × 22 $\mathrm{mm}$. Esta técnica pode ser utilizada também para a pesquisa de protozoários, para tanto pode ser empregado a este sedimento que restou no tubo uma solução de sulfato de zinco na densidade 1.180 , realizando a seguir 
a leitura do sobrenadante (KATAGIRI S e OLIVEIRA-SEQUEIRA TCG, 2010). O método Willis-Mollay é uma técnica qualitativa, utilizada geralmente para a visualização de ovos leves, para esta são utilizadas 2 a $5 \mathrm{~g}$ de fezes e aproximadamente $20 \mathrm{ml}$ de solução hipersaturada de $\mathrm{NaCl}$, esta solução pode ser colocada em um copo ou borrel (dentro de uma placa de Petri), completando-se estes recipientes com mais solução hipersaturada até formar um menisco e uma lâmina deve ser colocada sobre este copo ou borrel, por 10 a 15 minutos. Após este tempo a lâmina já pode ser examinada (HOFFMAN PR,1987).

O método de Gordon e Whitlock modificado (OPG) é uma técnica de flutuação, quantitativa, ou seja, associada a contagem de ovos. Para esta foram utilizadas 2 gramas de fezes e $58 \mathrm{~mL}$ de solução hipersaturada de $\mathrm{NaCl}$, filtrada com o uso de uma peneira ou gaze, e com o auxilio de uma pipeta os dois compartimentos da câmara de McMaster foram preenchidos para a leitura e quantificação dos ovos. Nesta diluição o resultado será multiplicado por 100 (HOFFMAN RP, 1987). Para a visualização das lâminas, foi utilizado um microscópio óptico, manuseando as objetivas de 10 e 40x para os métodos qualitativos e de 10x para a técnica quantitativa.

O presente estudo aprovado pelo Comitê de Ética em Pesquisa da Universidade Federal do Piauí (UFPI), sob número de parecer 3.724.219 e CAAE: 22935319.1.0000.5214, cumprindo com todos os aspectos legais e éticos previstos na Resolução 196/96, do Conselho Nacional de Saúde O propósito da pesquisa e a forma de participação foram explicados aos participantes, e os doadores das fezes que concordaram em participar da pesquisa assinaram o Termo de Consentimento Livre e Esclarecido (TCLE) autorizando a utilização do material coletado para fins de pesquisa.

Os participantes que apresentaram parasitos em suas amostras de fezes foram orientados a buscarem atendimento médico nos serviços de saúde disponíveis em suas comunidades, para que o tratamento fosse realizado adequadamente.

\section{RESULTADOS}

Os voluntários da pesquisa apresentaram idades entre 21 e 70 anos, variando entre pessoas do sexo feminino e masculino, 81 amostras foram doadas por 33 usuários da UBS Mod. 45 e 60 amostras doadas pelos usuários da UBS Mod. 14, totalizando 141 amostras. Os ovos identificados neste estudo foram dos helmintos: Ascaris lumbricoides, Trichuris trichiura, Enterobius vermicularis, Schistosoma mansoni, Taenia sp. e ovos da família Ancylostomatidae.

Das 141 amostras analisadas utilizando o método Hoffman, Pons e Janer-adaptado por Katagiri S e Oliveira-Sequeira TCG (2010), 14,18\% amostras (20) estavam positivas para ovos de helmintos intestinais e 85,82\% (121) negativas. Entre os ovos de parasitos encontrados: 6,38\% (9) correspondem a ovos do nematódeo Ascaris lumbricoides; 4,25\% (6), ovos de Trichuris trichiura; 2,13\% (3) ovos de Enterobius vermicularis e $1,42 \%$ (1) ovos de Taenia sp. (Tabela 1 ).

Tabela 1 - Prevalência de ovos de helmintos identificados em fezes de usuários de duas unidades básicas de saúde, da cidade de Parnaíba, Piauí, por meio do método de Hoffman, Pons e Janer-adaptado por Katagiri S e Oliveira-Sequeira TCG (2010).

\begin{tabular}{lcc}
\hline \multicolumn{1}{c}{ Parasitos identificados } & N (141) & Prevalência (\%) \\
\hline Ascaris lumbricoides & 9 & 6,38 \\
\hline Trichuris trichiura & 6 & 4,25 \\
\hline Enterobius vermicularis & 3 & 2,13 \\
\hline Taenia sp. & 2 & 1,42 \\
\hline
\end{tabular}

Fonte: Santos KR, et al., 2020.

Através do método de Willis-Mollay (Ferreira MU, 2012; Hoffman RP, 1987), foram analisadas 124 amostras, em 15,32\% (19) foram encontrados ovos de algum parasito e 84,68\% (105) das amostras estavam negativas. Em 5,64\% das amostras (7) foram encontrados ovos de Trichuris trichiura; em 4,84\% (6) ovos de 
Ascaris lumbricoides; em 2,42\% (3) das amostras ovos pertencentes à família Ancylostomatidae, em 1,62\% (2) ovos de Taenia sp. e em 0,81\% (1) das amostras ovos da espécie de Schistosoma mansoni (Tabela 2).

Tabela 2 - Prevalência de ovos de helmintos identificados em fezes de usuários de duas unidades básicas de saúde, da cidade de Parnaíba, Piauí, por meio do método de Willis-Mollay.

\begin{tabular}{lcc}
\hline \multicolumn{1}{c}{ Parasitos identificados } & N (124) & Prevalência (\%) \\
\hline Trichuris trichiura & 7 & 5,64 \\
\hline Ascaris lumbricoides & 6 & 4,84 \\
\hline Ancylostomatidae & 3 & 2,42 \\
\hline Taenia sp. & 2 & 1,62 \\
\hline Schistosoma mansoni & 1 & 0,81 \\
\hline
\end{tabular}

Fonte: Santos KR, et al., 2020.

Em relação ao método de Gordon e Whitlock-modificado (Hoffman RP, 1987), das 116 amostras analisadas, $12,94 \%$ (15) apresentaram ovos de alguma espécie de enteroparasito e 87,06\% (101) estavam negativas. Foram encontradas $6,89 \%$ (8) das amostras contendo ovos do nematódeo Trichuris trichiura e em $6,03 \%$ (7) ovos de Ascaris lumbricoides (Tabela 3).

Tabela 3 - Prevalência de ovos de helmintos identificados em fezes de usuários de duas unidades básicas de saúde, da cidade de Parnaíba, Piauí, por meio do método de Gordon e Whitlock-modificado.

\begin{tabular}{lcc}
\hline \multicolumn{1}{c}{ Parasitos identificados } & $\mathbf{N}(\mathbf{1 1 6})$ & Prevalência (\%) \\
\hline Trichuris trichiura & 8 & 6,89 \\
\hline Ascaris lumbricoides & 7 & 6,03 \\
\hline
\end{tabular}

Fonte: Santos KR, et al., 2020.

Em 2,42\% (3) das amostras analisadas pelo método de Willis-Mollay, foram observadas a presença de biparasitimos. Nos quais, as espécies encontradas em associação foram as seguintes: 1,62\% (2) das amostras continham ovos de Trichuris trichiura e ovos de Ascaris lumbricoides; e em 0,81\% (1) amostra continha ovos de Taenia sp. e ovos de Ascaris lumbricoides. Quanto à técnica Gordon e Whitlock-modificada, 2,59\% (2) das amostras também apresentaram biparasitismo, com associação entre Trichuris trichiura e Ascaris lumbricoides.

Os métodos de Hoffman, Pons e Janer-adaptado por Katagiri S e Oliveira-Sequeira TCG (2010) e de WillisMollay, permitiram a observação de uma maior prevalência de ovos de helmintos de famílias diferentes e pelo método de método de Gordon e Whitlock-modificado, foram identificados apenas ovos de duas espécies de nematódeos.

Quando comparando as três técnicas é possível observar que os métodos de Willis-Mollay recuperou ovos de ancilostomídeos e de Taenia sp. que não foram recuperados em nenhuma outra técnica, e pelo método de Hoffman, Pons e Janer-adaptado foram encontrados ovos do nematódeo Enterobius vermicularis. A maior prevalência de ovos do nematódeo Ascaris lumbricoides, 6,38\% (9), foi recuperado pelo método de Hoffman, Pons e Janer-adaptado, quando comparada aos outros métodos aqui utilizados (Gráfico 1). 
Gráfico 1 - Prevalência de ovos de helmintos identificados em fezes de usuários de duas unidades básicas de saúde, da cidade de Parnaíba, Piauí, por meio dos métodos Hoffman, Pons e Janer-adaptado por Katagiri S e Oliveira-Sequeira TCG (2010), Willis-Mollay e de Gordon e Whitlock-modificado.

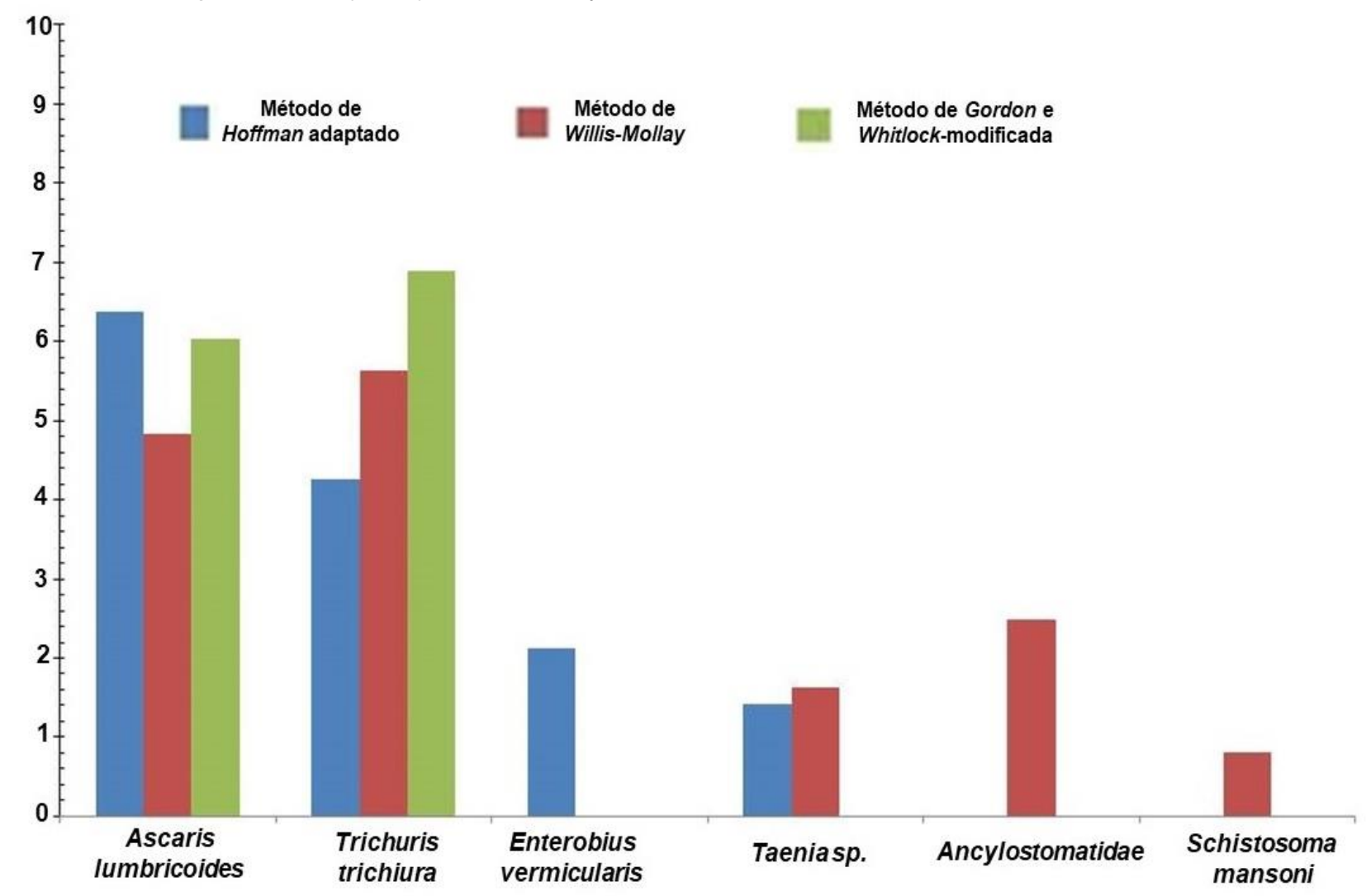

Fonte: Santos KR, et al., 2020.

\section{DISCUSSÃO}

As helmintíases estão entre as principais causas de problemas gastrintestinais em todo o mundo. A prevalência destes parasitos é alta em comunidades pobres de regiões tropicais, com condições socioeconômicas e higiênico-sanitárias precárias (ALEXANDRE TS, et al., 2015). O diagnóstico parasitológico das infecções causadas por estes enteroparasitos é significativo para o tratamento do paciente e controle da disseminação destes agentes (SILVA AO, et al., 2015). As três técnicas que foram empregadas neste estudo permitiram a detecção de diversos ovos de parasitos como: Ascaris lumbricoides, Trichuris trichiura, Enterobius vermicularis, Schistosoma mansoni, ovos da família Ancylostomatidae e os ovos de Taenia sp.

Os ovos do nematódeo Ascaris lumbricoides (6,38\%) foram encontrados em maior prevalência neste estudo, seguido de ovos de Trichuris trichiura (4,25\%) (Tabela 1, 2 e 3). Ambos são parasitos cuja forma de transmissão é pela via oral-fecal e fazem parte da lista das doenças negligenciadas. Ascaris lumbricoides é a espécie de nematódeo mais frequente encontrada nos estudos realizados no Brasil (SILVA JC, et al., 2011; CARDOSO AS, et al., 2018).

Em pesquisas anteriormente realizadas no município de Parnaíba, Piauí, foi observado uma maior prevalência de Ascaris lumbricoides (38,8\%) (Melo AFL, et al., 2014), ainda neste mesmo município, Alves FA et al. (2014) relatam que a prevalência relativa das enteroparasitoses foi de: $38 \%$ de pessoas infectadas por Ascaris lumbricoides e $20 \%$ por Ancilostomídeos.

Segundo Cardoso AS, et al. (2018) o helminto Ascaris lumbricoides, ocorre com maior frequência regiões tropicais e subtropicais, e as condições higiênico-sanitárias precárias colaboram para sua proliferação, estas justificativas corroboram com a alta prevalência deste nematódeo no presente estudo. Menezes RAO, et al. (2013) defende a importância da utilização de mais de um método em análises laboratoriais, para detectar as 
formas parasitárias de helmintos e protozoários, principalmente quando há uma baixa carga parasitária, pois, nenhum método é capaz de diagnosticar todos os parasitos.

Ou seja, o uso simultâneo de metodologias com diferentes sensibilidades para o diagnóstico, objetiva aumentar a eficiência do processo e a diminuição de resultados falso-negativos (SILVA NO e CAMELLO TCF, 2013). Neves DP (2010), também, defende a execução de vários métodos em cada amostra de fezes, sendo um método geral, um específico para larvas de helmintos e outro específico para cistos de protozoários. Entretanto, na maioria das vezes, tal procedimento se torna inviável, devido à quantidade de fezes que muitas vezes são insuficientes para a realização dos exames.

No presente estudo algumas amostras fornecidas pelos usuários das UBS foram insuficientes para realização das três metodologias propostas. A prioridade, neste estudo foi utilizar as amostras de fezes principalmente para a realização do método de Hoffman, Pons e Janer-adaptado por Katagiri S e OliveiraSequeira TCG (2010), por ser uma técnica menos usual em rotinas laboratoriais, o intuito era avaliarmos se essa adaptação nos auxiliaria a acelerar o procedimento, diminuindo o tempo de execução, e diminuindo a quantidade de detritos, mantendo a visibilidade dos parasitos. Devido esta insuficiência das amostras de fezes: 141 amostras foram analisadas pelo método de Hoffman, Pons e Janer-adaptado por Katagiri S e Oliveira-Sequeira TCG (2010) 124 amostras foram analisadas pela técnica de Willis-Mollay (Hoffman RP, 1987; Ferreira MU, 2012), e 116 amostras foram utilizadas para a realização da técnica de OPG (HOFFMAN RP, 1987).

Em nossas análises realizadas pelo método de Hoffman adaptado por Katagiri S e Oliveira-Sequeira TCG (2010) como já referido acima, observamos maior prevalência de ovos do nematódeo Ascaris lumbricoides corroborando com os achados de Seixas MTL, et al. (2012) que utilizaram o método convencional de Hoffman, Pons e Janer e encontraram $25 \%$ deste nematódeo. Seixas MTL, et al. (2012) relatam também o encontro de Strongyloides stercoralis em 2,5\% das amostras e Ancylostoma duodenale em 1,5\%.

Os resultados obtidos com a realização do método de Hoffman adaptado por Katagiri S, Oliveira-Sequeira TCG (2010), nos permitiu identificar ovos até mesmo do parasito Enterobius vermicularis com uma prevalência de 2,13\% (3). Este método não é específico para identificação do ovo deste parasito, segundo a literatura o método Graham (o da fita gomada ou fita adesiva) é o mais sensível para obtenção de ovos de parasitos do gênero Enterobius (FERREIRA MU, 2012; ZEIBIG EA, 2014).

No presente estudo, através da realização do método de Hoffman adaptado, foram identificados ovos do cestódeo Taenia spp. em 1,42\% (2) das análises. Este pode ter recuperado este cestódeo por ser um método de sedimentação e geralmente para o encontro deste parasito a técnica de Hoffman convecional consegue recuperá-la. A teníase é considera um importante problema de saúde pública, que pode ser adquirida através da ingestão dos ovos do parasito em água ou alimentos (BELLOTO MVT, et al., 2011).

No presente estudo quando empregamos a técnica de Willis Mollay, além da presença de ovos do parasito da família Ancylostomatidae em 2,42\% (3), foram encontrados também ovos do helminto Trichuris trichiura em 5,64\% (7) e Taenia sp. em 1,62\% (2), esta é uma técnica utilizada para a pesquisa de ovos leves de helmintos, e mesmo assim foi foram recuperados ovos de Ascaris lumbricoides com prevalência de 4,84\% (6) e $0,81 \%$ (um) de ovos de Schistosoma mansoni.

Segundo Ferreira UM (2012) a técnica de Willis é preconizada para recuperar ancilostomídeos, porém para o diagnóstico do parasito Schistosoma mansoni a técnica de Hoffman, Pons e Janer convencional é mais indicada, sendo este fato evidenciado por Furtado LFV e Melo ACFL (2011) que encontrou uma prevalência de $0,80 \%$ do nematódeo Schistosoma mansoni em uma população geronte da cidade de Parnaíba Piauí, utilizando a técnica de Hoffman convencional, a prevalência foi semelhante à encontrada em nosso estudo.

A técnica de Gordon e Whitlock modificado recuperou uma menor quantidade de parasitos dentre as amostras, quando comparado com as outras técnicas, demonstrando que esta não seria uma técnica indicada para a recuperação de parasitos em uma rotina laboratorial, ela só seria indicada caso o laboratorista ou geralmente um pesquisador, tivesse intenção de quantificar os ovos dos parasitas dentro das amostras, pois esta é menos dispendiosa, uma vez que para a realização da mesma há a necessidade apenas da Câmara

REAS/EJCH | Vol.Sup.n.52 | e3521 | DOI: https://doi.org/10.25248/reas.e3521.2020 Página 7 de 9 
de MacMaster, que é barata e de solução hipersaturada de NACL, também de baixo custo, sendo uma alternativa para a técnica de Kato-Katz, que é a técnica quantitativa específica para a quantificação dos ovos das fezes de seres humanos, porém que necessita de kits e reagentes que poderiam encarecer a análise (HOFFMAN RP, 1987; FERREIRA UM, 2012).

Em 2,42\% (3) das amostras analisadas pelo Método de Willis-Mollay, foi observada a presença de biparasitimos e em 97,58\% (121) monoparasitismo. Em relação à técnica de OPG, 1,72\% (2) das amostras também apresentaram biparasitismo e 98,28\% (114) de monoparasitismo. Estes corroboram com os encontrados em um estudo realizado com crianças no município de Parnaíba, Piauí, que também demonstra uma porcentagem maior de monoparasitismo (68\%), seguido do biparasitismo $(30 \%)$ e poliparasitismo $(3 \%)$ (ALVES FA, et al., 2014).

Maia CVA, et al. (2014) encontraram resultados relacionados aos diferentes ovos de parasitos encontrados em pessoas da região de Limoeiro do Norte, Ceará, semelhantes ao desta pesquisa, estes observaram que 86,6\% das amostras analisadas por eles apresentaram 9,7\% de monoparasitismo e biparasitismo e 3,6\% de poliparasitismo. Alves FA (2014) e Maia CVA, et al. (2014) encontraram mais de dois parasitos (poliparasitismo) infectando um só indivíduo o que não foi observado no presente estudo.

Os resultados negativos para as três metodologias utilizadas, foram superiores aos resultados positivos. Uma justificativa foi as unidades básicas terem promovido dias antes deste estudo a desverminação de $90,07 \%$ (127) dos usuários, estes receberam drogas antiparasitárias, conforme relataram durante o preenchimento das fichas individuais. Os medicamentos mais utilizados foram o Albendazol $62,93 \%$ (80), seguido do Mebendazol 37,03\% (47). Estas são as principais drogas utilizadas no tratamento das parasitoses intestinais, tais como: Ascaris lumbricoides, Strongyloides stercoralis, ancilostomídeos, Enterobius vermicularis e Trichuris trichiura. Lembrando que, nenhum destes medicamentos leva a cura completa, daí a importância da realização dos exames coproparasitológicos para a distinção dos parasitos e utilização da medicação específica para os diferentes parasitos (ANDRADE EC, et al., 2010).

\section{CONCLUSÃO}

Os métodos propostos neste estudo demonstraram serem ótimas alternativas para pesquisa de parasitos intestinais. O método de Willis-Mollay, recuperou um número maior de gêneros de parasitos e demonstrou ser mais rápido e de fácil execução, apresentando boa visibilidade dos ovos de helmintos intestinais. Entretanto caso haja a necessidade de se pesquisar também protozoários, o método de Hoffman, Pons e Janer-adaptado por Katagiri S e Oliveira-Sequeira TCG (2010) seria uma ótima opção, uma vez que possibilitou o encontro de quase todos os parasitos que foram detectados pelo método de Willis e com a vantagem de poder ser adaptado para a realização da técnica de centrífugo-flutuação com sulfato de zinco, utilizando o sedimento restante. Com a realização das três técnicas coproparasitológicas, pode-se observar que as espécies de Ascaris lumbricoides e Trichuris trichiura foram às de maior prevalência na população estudada. O emprego de mais de um método para o diagnóstico parasitológico é recomendado para que se tenha um resultado de qualidade, uma vez que, cada metodologia empregada é especifica para um determinado parasito.

\section{REFERÊNCIAS}

1. ALEXANDRE TS, et al. Prevalência de protozoários intestinais em escolares de uma Unidade De Ensino da Rede Pública do Município de Vitorino Freire - MA. Revista Científica do ITPAC. 2015; 8(2): 4-12.

2. ALVES FA, et al. Aspectos epidemiológicos das enteroparasitoses em crianças domiciliadas em um assentamento rural no nordeste brasileiro. Revista Eletrônica Acervo Saúde. 2014; 6(3): 666-676.

3. ANDRADE EC, et al. Cesca MG. Parasitoses intestinais: uma revisão sobre seus aspectos sociais, epidemiológicos, clínicos e terapêuticos. Revista Atenção Primaria à Saúde. 2010; 13(2): 231-240.

4. BELLOTO MVT, et al. Enteroparasitoses numa população de escolares da rede pública de ensino do Município de Mirassol, São Paulo, Brasil. Revista Pan-Amazônica de Saúde. 2011; 2(1):37-44.

5. BELO VS, et al. Fatores associados à ocorrência de parasitoses intestinais em uma população de crianças e adolescentes. Revista Paulista de Pediatria. 2012; 30(2):195-201. 
6. CARDOSO, AS et al. Ocorrência de Ascaris lumbricoides em crianças do Estado do Maranhão. Brazilian Journal of Surgery and Clinical Research - BJSCR. 2018; 23(3): p.20-25.

7. CERQUEIRA EJL, et al. Análise comparativa da Sensibilidade da Técnica de Willis no diagnóstico parasitológico da ancilostomíase. Diálogos e Ciência. 2007; (5):1-7.

8. CHIEFFI PP, et al. Avaliação da eficácia do método de kato-katz no diagnóstico parasitológico da esquistossomose mansónica. Revista do Instituto Adolfo Lutz, 1981; 41(1): 23-30.

9. FERNANDES FC, BARSOSA FH. Ocorrência de parasitoses intestinais entre crianças da creche Menino Jesus do município de Dores do Indaiá, Minas Gerais. Revista Ciência Equatorial. 2011;1(1):29-37.

10. Furtado LFV, Melo ACFL. Prevalência e aspectos epidemiológicos de enteroparasitoses na população geronte de Parnaíba, Estado do Piauí. Revista da Sociedade Brasileira de Medicina Tropical. 2011; 44 (4): 513-515.

11. FERREIRA MU. Parasitologia Contemporânea. 1. ed. Rio de Janeiro: Guanabara Koogan, 2012; 236p.

12. GONÇALVES GS, et al. Estudo comparativo de técnicas parasitológicas de sedimentação espontânea: Kit comercial Coproplus $® 10$ e método de Hoffman, Pons e Janer - HPJ. Revista Iniciação Científica. 2016; 16(4): 124 -129.

13. GRISOLIO APR, et al. Comportamento de cães e gatos: sua importância para a saúde pública. Revista de Ciência Veterinária e Saúde Pública. 2017; 4 (1): 117-126.

14. HOFFMAN, R. P. Diagnóstico de parasitismo veterinário. 1ed. Porto Alegre, RS, Sulina. 1987; 156p.

15. IBGE - Instituto Brasileiro de Geografia e Estatística. Parnaíba - população estimada 2015.

16. IBGE - Instituto Brasileiro de Geografia e Estatística. Parnaíba - população estimada 2010.

17. KATAGIRI S, OLIVEIRA-SEQUEIRA, TCG. Comparison of three concentration methods for the recovery of canine intestinal parasites from stool samples. Experimental Parasitology. San Diego: Academic Press Inc. Elsevier B.V. 2010; 126 (2): 214-216.

18. LABRUNA M.B, et al. Prevalência de endoparasitas em cães da área urbana do município de monte negro, Rondônia. Arquivos do Instituto Biológico, 2006; 73 (2): 183-193.

19. MAIA CVA, et al. Fatores sociossanitários e parasitoses intestinais em Limoeiro do Norte, CE. Revista Brasileira de Geografia Médica e da Saúde. 2014; 10(19): 50 - 64.

20. MELO AFL, et al. Aspectos Epidemiológicos das Enteroparasitoses em Crianças de uma Unidade Pública de Ensino de Parnaíba, Piauí. UNOPAR Cient. Ciênc. Biol. Saúde. 2014; 3(16): 191-196.

21. MENEZES RAO, et al. Sensibilidade de métodos parasitológicos para o diagnóstico das enteroparasitoses em Macapá - Amapá, Brasil. Revista de Biologia e Ciências da Terra. 2013; 13(1): 66-73.

22. MONTEIRO, SG. Parasitologia veterinária. 1 ed. São Paulo: Roca, 2010; 356p.

23. NEVES DP. Parasitologia humana. 11 ed. São Paulo: Atheneu, 2010; 498p.

24. NUNES XP, et al. Anemia ferropriva, enteroparasitoses e esgotamento sanitário. Revista Brasileira de Pesquisa em Saúde. 2014; 16 (1): 118-124.

25. SANTOS KR, et al. Análise parasitológica de ambientes utilizados para recreação infantil. Enciclopédia Biosfera. 2019; 16 (29): $1992-1994$.

26. SEIXAS MTL, et al. Avaliação da frequência de parasitos intestinais e do estado nutricional em escolares de uma área periurbana de Salvador, Bahia, Brasil. Revista de Patologia Tropical. 2012; 40(4): 304-14.

27. SILVA JC, et al. Parasitismo por Ascaris lumbricoides e seus aspectos epidemiológicos em crianças do Estado do Maranhão Revista da Sociedade Brasileira de Medicina Tropical. 2011; 44(1): 100-102.

28. SILVA NO, CAMELLO TCF. Avaliação da presença de protozoários intestinais em hospital universitário do Rio de Janeiro, Brasil. Revista de Saúde e Educação. 2013; 1(1): 3-9.

29. SILVA AO, et al. Epidemiologia e prevenção de parasitoses intestinais em crianças das creches municipais de Itapuranga-GO. Revista Faculdade Montes Belos. 2015; 8 (2): 1-17.

30. SOUSA RA, et al. Utilização de peças teatrais como auxílio na prevenção de parasitoses. Revista Ciência em Extensão. 2015; 11 (1): 139-147.

31. VASCONCELOS IAB, et al. Prevalência de parasitoses intestinais entre crianças de 4-12 anos no Crato, estado do Ceará: Um problema recorrente de saúde publica. Fortaleza (CE, Brasil). Acta Scientiarum Health Sciences. 2011; 33(1): 35-41.

32. ZEIBIG EA. Parasitologia Clínica: uma abordagem clínico-laboratorial. 1 ed. Rio de Janeiro: Elsevier, 2014; 392p. 